\title{
Cytoskeleton-associated protein 4 is a novel serodiagnostic marker for esophageal squamous-cell carcinoma
}

This article was published in the following Dove Press journal:

OncoTargets and Therapy

\author{
Lei Chen ${ }^{1,2}$ \\ Chuanwen You ${ }^{1,2}$ \\ Xiaowei Jin ${ }^{1,2}$ \\ Lei Zhou ${ }^{1,2}$ \\ Liyou Huang ${ }^{1,2}$ \\ Yanhua Wang ${ }^{1,2}$ \\ 'Department of Oncology, Suqian \\ People's Hospital, Nanjing Drum \\ Tower Hospital Group, Suqian, China; \\ ${ }^{2}$ Department of Oncology, Suqian \\ Affiliated Hospital of Xuzhou Medical \\ University, Suqian, China
}

Background: Recent years have witnessed significant progress in the treatment of esophageal squamous-cell carcinoma (ESCC); however, the prognosis of ESCC is still unsatisfactory. Biomarkers are required to improve identification of high-risk populations and help management of ESCC. This study was to evaluate the role of serum CKAP4 in ESCC.

Methods: This longitudinal study recruited 207 ESCC patients and age-/sex-matched healthy controls. Circulating levels of CKAP4 were measured using ELISA kits, while the expression of CKAP4 in esophageal tissue was evaluated using Western blotting.

Results: Serum CKAP4 levels were higher in ESCC patients $(380.2 \pm 171.3 \mathrm{pg} / \mathrm{mL})$ than healthy controls $(271.8 \pm 97.4 \mathrm{pg} / \mathrm{mL} ; P<0.001)$. The area under the receiver-operating characteristic curve of serum CKAP4 levels to identify the presence of ESCC was $0.675(95 \% \mathrm{CI}$ $0.622-0.728 ; P<0.001)$. According to Youden's index, the best cutoff value was $429.1 \mathrm{pg} / \mathrm{mL}$ (sensitivity 0.415 and specificity 0.995 ). Furthermore, after follow-up, multivariate analyses identified that pathological lymph node metastases were the poorest prognostic factor (HR 1.862, 95\% CI 1.093-3.173; $P=0.022$ ), followed by serum CKAP4 (HR 1.437, 95\% CI 1.025-2.014; $P=0.035$ ). When stratified by tertiles of serum CKAP4, subjects in the first tertile presented a mean survival time of 75.4 months (95\% CI 68.0-81.9), which decreased significantly in the second tertile (73.8 months, $95 \%$ CI $61.4-86.3)$ and the third tertile (59.9 months, $95 \%$ CI 49.8-70.0, log-rank $\left.\chi^{2}=8.235 ; P=0.016\right)$.

Conclusion: These results suggested that serum CKAP4 could be a potential biomarker for clinical management of ESCC.

Keywords: esophageal squamous-cell carcinoma, biomarker, Dickkopf 1, cytoskeletonassociated protein 4

\section{Introduction}

Esophageal cancer is the eighth most common cancer and the sixth leading cause of cancer-related deaths worldwide and includes two histological types: esophageal adenocarcinoma and esophageal squamous-cell carcinoma (ESCC). ${ }^{1,2}$ The latter is the major histological type and the predominant subtype in East Asia. ${ }^{3}$ Even though recent years have witnessed significant progress in endoscopic therapy, chemotherapy, and radiation, the prognosis of ESCC is still unsatisfactory. ${ }^{4}$ Evaluation dependent on clinicopathological characteristics is still poor due to significant variability within the same stage..$^{5-7}$ Therefore, novel biological markers are required to improve accurate identification of high-risk populations and appropriate management of ESCC.

DKK1 is a secreted protein induced by the $\beta$-catenin-TCF4 complex. ${ }^{8}$ As a Wnt signal-negative regulator, DKK1 is highly expressed in various carcinomas.
Correspondence: Chuanwen You Department of Oncology, Suqian People's Hospital, Nanjing Drum Tower Hospita Group, I 38 Huanghe South Road, Sucheng, Suqian, Jiangsu 223800, China Tel +8652784239365 Fax +8652784239365 Email cwyou_sqph@163.com 
It has been reported that DKK1 is an independent and significant predictive factor of ESCC and associated with poor prognosis. ${ }^{8-10} \mathrm{CKAP} 4$, a $63 \mathrm{kDa}$ palmitoylated type II transmembrane protein, was originally discovered to anchor the endoplasmic reticulum to microtubules in epithelial cells. ${ }^{11-13}$

Recent evidence supports CKAP4 being a novel receptor of DKK $1 .{ }^{10,14}$ At the plasma membrane, the binding of DKK1 to CKAP4 triggers the PI3K-Akt pathway, which promotes the proliferation of various carcinoma cell culture lines. ${ }^{10,14}$ Previous findings have suggested that CKAP4 might be a novel molecular target for the clinical management of cancer.

Li et $\mathrm{al}^{15}$ found increased expression of CKAP4 in intrahepatic cholangiocellular carcinoma patients. Besides, CKAP4 presented associations with tumor size, metastasis situation, and TNM stages. The study indicated that CKAP4 was an independent predictor for overall survival, which suggested that it was a prognostic marker of intrahepatic cholangiocellular carcinoma. Shinno et $\mathrm{al}^{10}$ found that compared with normal esophageal mucosa, DKK1 and CKAP4 had high expression in regions of ESCC by immunohistochemical analyses. Moreover, expression of DKK1 and CKAP4 was associated with poor prognosis and relapse-free survival.

Current evidence supports that tumor expression of CKAP4 in several carcinomas, evaluated by immunohistochemistry, has an association with cancer development and prognosis and thus may be a novel marker for cancers. ${ }^{10,12}$ Recently, Yanagita et al ${ }^{11}$ confirmed that CKAP4 was a secreted protein produced by lung cancer cells. They found that serum CKAP levels were higher in lung cancer patients than in healthy controls. Also, serum CKAP4 levels correlated with distant metastasis. Furthermore, the sensitivity of serum CKAP4 was higher than that of other markers for lung cancer. This study suggested that CKAP4 might be a serodiagnostic marker for lung cancer.

In this study, first, we measured the serum levels of CKAP4 in 207 ESCC patients and 207 age-/sex-matched healthy controls using commercial ELISA kits. Also, the association between serum CKAP4 and disease-free survival was evaluated.

\section{Methods}

\section{Study design and patients}

This longitudinal cohort study recruited 207 ESCC patients who underwent surgical resection at Suqian People's Hospital between 2011 and 2016. Subjects with severe cardiopulmonary disease, severe liver diseases, renal insufficiency, and endocrine diseases were excluded. Subtotal esophagectomy via thoracotomy with lymphadenectomy was performed in the patients. None of them died due to postoperative complications. After surgery, patients were surveyed by physical and biochemical examinations semiannually and computed tomography and endoscopy annually until tumor recurrence was evident. If their systemic conditions permitted, ESCC patients with tumor recurrence received chemotherapy or chemoradiotherapy. A total of 207 healthy subjects who attended annual endoscopic examination in the outpatient department were invited to participate in this study on the principle of age-/sex-matched case-control. The project was approved by the Ethics Committee of Suqian People's Hospital in accordance with the Helsinki Declaration of 1964. Before participation, each participant gave written informed consent.

\section{Examinations}

Histopathologically confirmed ESCC tissue samples were chosen for Western blotting, while normal or inflammatory tissue samples from healthy participants were grouped as controls. Rabbit monoclonal anti-CKAP4 (ab84712) antibody was purchased from Abcam (Cambridge, UK). Blood samples were obtained and frozen for further evaluation of serum CKAP4 levels by ELISA (CSB-EL005453HU; Cusabio, Wuhan, China).

We randomly selected six participants (three ESCC patients and three healthy controls). We measured circulating levels of CKAP4 with ELISA and evaluated CKAP4 expression in normal or carcinomatous esophagus tissues by Western blotting. Results showed reliable consistency between circulating levels and tissue expression of CKAP4 in regions of interest, as shown in Figure S1.

\section{Statistical methods}

Student's unpaired $t$-tests and $\chi^{2}$ tests were used for comparisons between two groups. Receiver-operating characteristic (ROC) curves for serum CKAP4 was developed to identify the presence of ESCC. Prognostic variables were evaluated by log-lank test, and disease-free survival was analyzed by Kaplan-Meier methods. Univariate and multivariate Cox proportional-hazard regression models were used to analyze prognostic variables for disease-free survival. All statistical analyses were performed by SPSS (version 21.0; IBM Corporation, Armonk, NY, USA). Two-sided $P<0.05$ was considered statistically significant.

\section{Results}

Table 1 presents the characteristics of ESCC patients in this study. 
Table I Patient characteristics

\begin{tabular}{|c|c|}
\hline Number & 207 \\
\hline Age (years) & $61.6 \pm 14.2$ \\
\hline Follow-up (months) & $31.0 \pm 20.9$ \\
\hline Serum CKAP4 (pg/mL) & $380.2 \pm 171.3$ \\
\hline Sex (male/female) & $144 / 63$ \\
\hline Histology (good/moderate/poor) & $33 / 139 / 35$ \\
\hline Location (upper/middle/lower) & $22 / 104 / 81$ \\
\hline Chemotherapy (no/yes) & $117 / 90$ \\
\hline pT (I-2/3-4) & $68 / 139$ \\
\hline $\mathrm{pN}(<4 / \geq 4)$ & $112 / 95$ \\
\hline pStage $(I / I / I I I / I V)$ & $23 / 64 / 83 / 37$ \\
\hline
\end{tabular}

Note: Data are represented as mean $\pm \mathrm{SD}$ for continuous variables. $\mathrm{pN}, \mathrm{pT}$, and pStage according to TNM classification.

\section{Serum levels of CKAP4}

As shown in Figure 1, serum CKAP4 levels were higher in ESCC patients $(\mathrm{n}=207,380.2 \pm 171.3 \mathrm{pg} / \mathrm{mL})$ than healthy controls ( $\mathrm{n}=207,271.8 \pm 97.4 \mathrm{pg} / \mathrm{mL} ; P<0.001)$.

\section{Performance of CKAP4 in diagnosis of ESCC}

Figure 2 shows the ROC curve of serum CKAP4 levels to identify the presence of ESCC (area under curve 0.675 [95\% CI 0.622-0.728, $P<0.001]$ ). According to Youden's index, the best cutoff value was $429.1 \mathrm{pg} / \mathrm{mL}$ (sensitivity 0.415 and specificity 0.995$)$.

\section{Correlation between CKAP4 levels and survival}

As indicated in Table 2, on univariate analysis, relationships among histology (HR 0.630, 95\% CI 1.713-5.059; $P=0.045$ ), pT (HR 2.135, 95\% CI 1.132-4.025; $P=0.019), \mathrm{pN}(\mathrm{HR}$

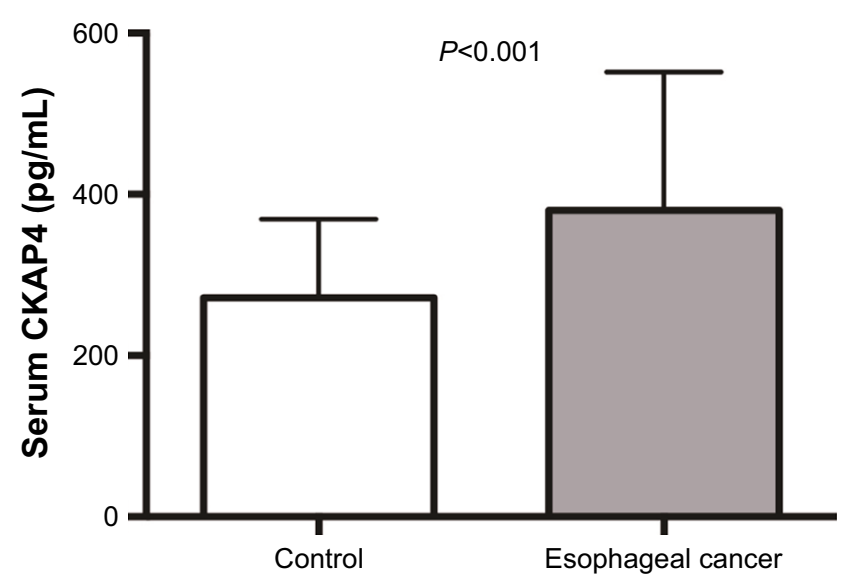

Figure I Comparison of serum CKAP4 between healthy controls and cancer cases.

Note: Serum CKAP4 levels between healthy controls $(\mathrm{n}=207,271.8 \pm 97.4 \mathrm{pg} / \mathrm{mL})$ and esophageal cancer patients $(\mathrm{n}=207,380.2 \pm \mathrm{I} 7 \mathrm{I} .3 \mathrm{pg} / \mathrm{mL} ; \mathrm{P}<0.00 \mathrm{I})$.

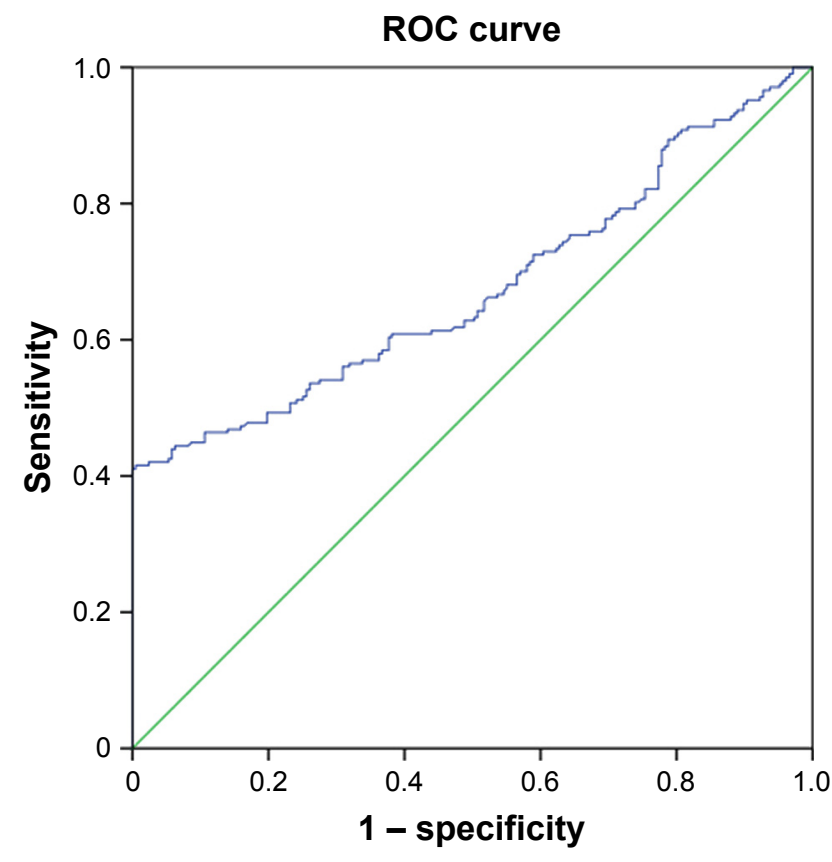

Figure 2 ROC curve of CKAP4 to identify esophageal cancer.

Notes: ROC curve of serum CKAP4 levels to identify the presence of esophageal cancer (area under ROC curve, 0.675; 95\% Cl 0.622-0.728; $P<0.00 \mathrm{I}$ ). According to the Youden's index, the best cutoff value was $429.1 \mathrm{pg} / \mathrm{mL}$ (sensitivity, 0.415 ; specificity, 0.995; positive predictive value, 0.989 ; negative predictive value, 0.630 ). Diagonal segments are produced by ties.

Abbreviation: ROC, receiver-operating characteristic.

2.112, 95\% CI 1.250-3.569; $P=0.005)$, serum CKAP4 (HR $1.546,95 \%$ CI 1.105-2.162; $P=0.011)$, and disease-free survival were significant.

Table 3 presents multivariate analysis using the four statistically significant variables from univariate analysis, identifying that pathological lymph node-metastases were the poorest prognostic factor (HR 1.862, 95\% CI 1.093-3.173; $P=0.022$ ), followed by serum CKAP4 (HR 1.437, 95\% CI 1.025-2.014; $P=0.035$ ).

Table 4 shows mean survival time (75.0 months, 95\% CI 68.0-81.9). When stratified by tertiles of serum CKAP4, subjects in the first tertile presented a mean survival time

Table 2 Univariate survival analysis of disease-free survival by Cox proportional-hazard model

\begin{tabular}{l|l|l|l}
\hline & HR & $\mathbf{9 5 \%} \mathbf{C l}$ & P-value \\
\hline Age & 0.758 & $0.453-1.267$ & 0.290 \\
Sex & 1.138 & $0.646-2.003$ & 0.655 \\
Histology & 0.630 & $0.402-0.989$ & 0.045 \\
$\quad$ Location & 0.748 & $0.496-1.126$ & 0.164 \\
$\quad$ Chemotherapy & 0.596 & $0.348-1.022$ & 0.060 \\
PT & 2.135 & $1.132-4.025$ & 0.019 \\
PN & 2.112 & $1.250-3.569$ & 0.005 \\
CKAP4 & 1.546 & $1.105-2.162$ & 0.011 \\
\hline
\end{tabular}

Note: Histology (good/moderate/poor), location (upper/middle/lower), chemotherapy (no/yes), $\mathrm{pT}(I-2 / 3-4)$, and $\mathrm{pN}(<4 / \geq 4)$ based on TNM classification. 
Table 3 Multivariate analysis of disease-free survival by Cox proportional-hazard model

\begin{tabular}{l|l|l|l}
\hline & HR & $95 \% \mathbf{C l}$ & P-value \\
\hline Histology & 0.686 & $0.435-1.080$ & 0.104 \\
PT & 1.835 & $0.962-3.499$ & 0.065 \\
PN & 1.862 & $1.093-3.173$ & 0.022 \\
CKAP4 & 1.437 & $1.025-2.014$ & 0.035 \\
\hline
\end{tabular}

Note: Histology (good/moderate/poor), pT $(I-2 / 3-4)$, and pN $(<4 / \geq 4)$ based on the TNM classification.

of 75.4 months (95\% CI 68.0-81.9), which was significantly decreased in the second tertile (73.8 months, 95\% CI 61.4-86.3) and the third tertile (59.9 months, 95\% CI 49.8-70.0, log-rank [Mantel-Cox] $\chi^{2}=8.235$; $P=0.016$; Figure 3).

\section{Discussion}

Inspired by the studies of Shinno et $\mathrm{al}^{10}$ and Yanagita et al, ${ }^{11}$ we first evaluated serum levels of CKAP4 in cancer patients. We found that serum CKAP4 levels were higher in ESCC patients than healthy controls. Besides, serum CKAP4 presented fine specificity to identify the presence of ESCC. Furthermore, circulating levels of CKAP4 were associated with disease-free survival time.

Recent research has reported that the expression of CKAP4 presented associations with clinical outcome and prognosis of intrahepatic cholangiocarcinoma and hepatocellular carcinoma. ${ }^{10,15}$ Shinno et a $1^{10}$ demonstrated that DKK1 activates Akt via CKAP4 in ESCC cells. The PI3K-Akt pathway might be a downstream cascade of the DKK1CKAP4 signaling axis in ESCC cases, which is positive for both proteins. Reports have shown that about $40 \%$ of ESCC patients and $60 \%$ pancreatic and non-small-cell lung cancer patients showed overexpression of CKAP4. ${ }^{10,14}$ The role and mechanisms of CKAP4 in carcinomas are still unknown. Further investigation is needed for better understanding of CKAP4 in esophageal cancer.

Despite recent progress in clinical management and treatment, ESCC patients' survival is still poor. ${ }^{10,16}$ Given that most patients are initially diagnosed in advanced stages, practical biomarkers for identifying patients at resectable

Table 4 Survival time by CKAP4 tertiles

\begin{tabular}{l|l|l}
\hline & Mean (months) & $\mathbf{9 5 \%} \mathbf{C l}$ \\
\hline TI & 75.4 & $67.6-83.1$ \\
T2 & 73.8 & $61.4-86.3$ \\
T3 & 59.9 & $49.8-70.0$ \\
Overall & 75.0 & $68.0-81.9$ \\
\hline
\end{tabular}

Notes: Disease-free survival according to serum CKAP4 tertile. Log-rank (MantelCox): $\chi^{2}=8.235, P=0.016$.

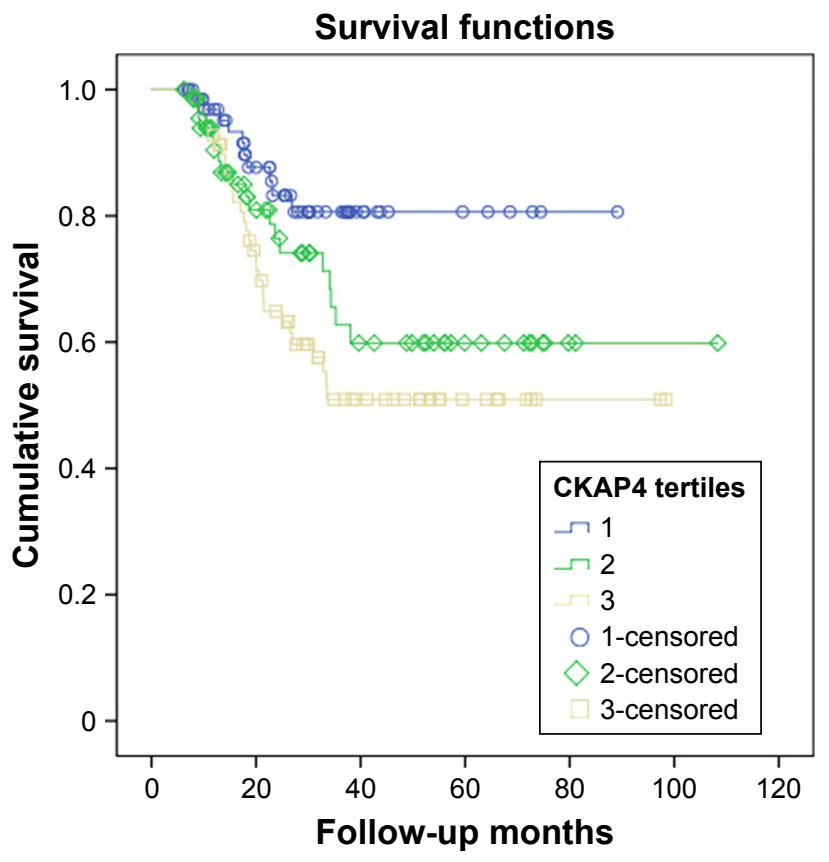

Figure 3 Survival curves according to serum CKAP4 levels.

Note: Disease-free-survival curve classified according to the tertiles of serum CKAP4 levels for all patients, plotted by Kaplan-Meier methods.

early stages of ESCC are urgently required. It has been reported that CKAP4 was secreted through exosomes of colon and ovarian cancer cells and detected in urine. ${ }^{17-19}$ Yanagita et $\mathrm{a}^{11}$ generated an antibody named KU-Lu-1 by reverse-phase protein array, which could recognize CKAP4. They demonstrated serum CKAP4 as an early serodiagnostic marker for lung cancer.

Inspired by previous findings, we tried to evaluate serum levels of CKAP4 in ESCC patients using commercial ELISA kits. To begin with, we validated circulating levels of CKAP4 and their association with tissue expression of CKAP4 in ESCC, which presented reliable consistency. Then, we found that serum CKAP4 levels increased in ESCC patients compared with healthy controls. Also, serum CKAP4 presented high specificity to identify the presence of ESCC, even though sensitivity was low. This also indicated an acceptable negative predictive value and a high positive predictive value. In a hospital-based case-control design, cross-sectional studies in multiple endoscopic centers are required to evaluate the utility of serum CKAP4 measurement in screening ESCC in symptomatic populations. We believe that CKAP4 could be utilized as a biomarker in endoscopic examination and help endoscopists to screen symptomatic populations and identify ESCC patients. A total of 207 ESCC patients who underwent surgical resection were followed up. Imaging/pathology information and treatment procedures were recorded. Circulating CKAP4 levels were associated 
with disease-free survival, which suggested serum CKAP4 might also be a biomarker for clinical prognosis of ESCC.

Our study has some limitations. First, given the close association between DKK 1 and CKAP $4,{ }^{20}$ further studies on serum levels of DKK1 are required. Second, comparisons with other biomarkers for ESCC, eg, SCC antigen, ${ }^{21}$ are needed. Meanwhile, patients with other esophageal diseases, eg, esophageal polyps, should be included to rule out potential false-positive results. Moreover, experiments should be performed to discover the secretion process of CKAP4 protein from carcinoma tissue. Finally, the mechanism of increased CKAP4 expression and its association with poor prognosis needs further investigation.

\section{Conclusion}

This study found that serum CKAP4 levels were higher in ESCC patients than healthy controls. Circulating levels of CKAP4 were associated with disease-free survival. This suggests that serum CKAP4 could be a potential biomarker for the clinical management of ESCC.

\section{Acknowledgment}

This study was funded by Suqian Projects of Science and Technology (Z2018078).

\section{Disclosure}

The authors report no conflicts of interest in this work.

\section{References}

1. Pennathur A, Gibson MK, Jobe BA, Luketich JD. Oesophageal carcinoma. Lancet. 2013;381(9864):400-412.

2. Ferlay J, Soerjomataram I, Dikshit R, et al. Cancer incidence and mortality worldwide: sources, methods and major patterns in GLOBOCAN 2012. Int J Cancer. 2015;136(5):E359-E386.

3. Ohashi S, Miyamoto S, Kikuchi O, Goto T, Amanuma Y, Muto M. Recent advances from basic and clinical studies of esophageal squamous cell carcinoma. Gastroenterology. 2015;149(7):1700-1715.

4. Tamoto E, Tada M, Murakawa K, et al. Gene-expression profile changes correlated with tumor progression and lymph node metastasis in esophageal cancer. Clin Cancer Res. 2004;10(11):3629-3638.

5. Sakaeda T, Yamamori M, Kuwahara A, Nishiguchi K. Pharmacokinetics and pharmacogenomics in esophageal cancer chemoradiotherapy. Adv Drug Deliv Rev. 2009;61(5):388-401.
6. Wang D, Plukker JTM, Coppes RP. Cancer stem cells with increased metastatic potential as a therapeutic target for esophageal cancer. Semin Cancer Biol. 2017;44:60-66.

7. Thallinger CM, Raderer M, Hejna M. Esophageal cancer: a critical evaluation of systemic second-line therapy. J Clin Oncol. 2011;29(35): 4709-4714.

8. Niehrs C. Function and biological roles of the Dickkopf family of Wnt modulators. Oncogene. 2006;25(57):7469-7481.

9. Makino T, Yamasaki M, Takemasa I, et al. Dickkopf-1 expression as a marker for predicting clinical outcome in esophageal squamous cell carcinoma. Ann Surg Oncol. 2009;16(7):2058-2064.

10. Shinno N, Kimura H, Sada R, et al. Activation of the Dickkopf1-CKAP4 pathway is associated with poor prognosis of esophageal cancer and anti-CKAP4 antibody may be a new therapeutic drug. Oncogene. 2018; 37(26):3471-3484.

11. Yanagita K, Nagashio R, Jiang S-X, et al. Cytoskeleton-Associated Protein 4 Is a Novel Serodiagnostic Marker for Lung Cancer. Am J Pathol. 2018;188(6):1328-1333.

12. Kikuchi A, Fumoto K, Kimura H. The Dickkopf1-cytoskeletonassociated protein 4 axis creates a novel signalling pathway and may represent a molecular target for cancer therapy. Br J Pharmacol. 2017; 174(24):4651-4665.

13. Gladka MM, Molenaar B, de Ruiter H, et al. Single-Cell Sequencing of the Healthy and Diseased Heart Reveals Cytoskeleton-Associated Protein 4 as a New Modulator of Fibroblasts Activation. Circulation. 2018;138(2):166-180.

14. Kimura H, Fumoto K, Shojima K, et al. CKAP4 is a Dickkopf1 receptor and is involved in tumor progression. J Clin Invest. 2016;126(7): 2689-2705.

15. Li MH, Dong LW, Li SX, et al. Expression of cytoskeleton-associated protein 4 is related to lymphatic metastasis and indicates prognosis of intrahepatic cholangiocarcinoma patients after surgery resection. Cancer Lett. 2013;337(2):248-253.

16. Visser E, Franken IA, Brosens LA, Ruurda JP, van Hillegersberg R. Prognostic gene expression profiling in esophageal cancer: a systematic review. Oncotarget. 2017;8(3):5566-5577.

17. Demory Beckler M, Higginbotham JN, Franklin JL, et al. Proteomic analysis of exosomes from mutant KRAS colon cancer cells identifies intercellular transfer of mutant KRAS. Mol Cell Proteomics. 2013; 12(2):343-355.

18. Liang B, Peng P, Chen S, et al. Characterization and proteomic analysis of ovarian cancer-derived exosomes. J Proteomics. 2013;80:171-182.

19. Gonzales PA, Pisitkun T, Hoffert JD, et al. Large-scale proteomics and phosphoproteomics of urinary exosomes. J Am Soc Nephrol. 2009; 20(2):363-379.

20. Razzaq TM, Bass R, Vines DJ, Werner F, Whawell SA, Ellis V. Functional regulation of tissue plasminogen activator on the surface of vascular smooth muscle cells by the type-II transmembrane protein p63 (CKAP4). J Biol Chem. 2003;278(43):42679-42685.

21. Charakorn C, Thadanipon K, Chaijindaratana S, Rattanasiri S, Numthavaj $\mathrm{P}$, Thakkinstian A. The association between serum squamous cell carcinoma antigen and recurrence and survival of patients with cervical squamous cell carcinoma: a systematic review and metaanalysis. Gynecol Oncol. 2018;150(1):190-200. 


\section{Supplementary material}

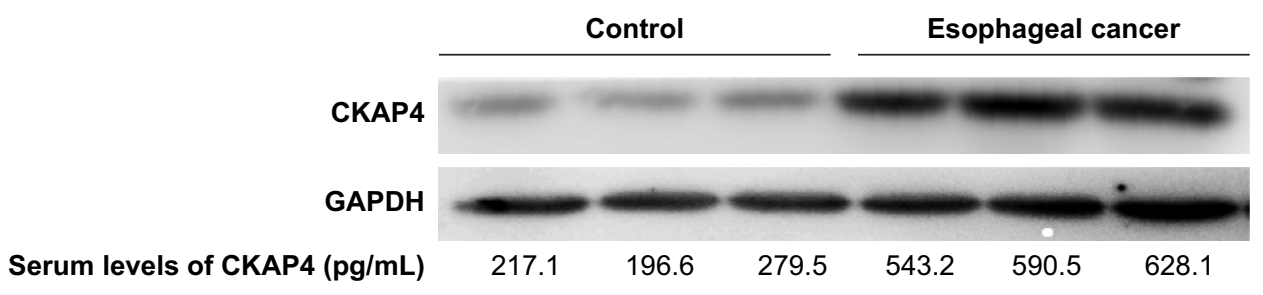

Figure SI Six participants (three ESCC patients and three healthy controls) were selected.

Notes: We measured circulating levels of CKAP4 using ELISA and evaluated CKAP4 expression in esophageal normal or carcinomatous tissue using Western blotting. Results presented reliable consistency between circulating levels and tissue expression of CKAP4 in regions of interest.

Abbreviation: ESCC, esophageal squamous-cell carcinoma.

\section{Publish your work in this journal}

OncoTargets and Therapy is an international, peer-reviewed, open access journal focusing on the pathological basis of all cancers, potential targets for therapy and treatment protocols employed to improve the management of cancer patients. The journal also focuses on the impact of management programs and new therapeutic agents and protocols on

patient perspectives such as quality of life, adherence and satisfaction. The manuscript management system is completely online and includes a very quick and fair peer-review system, which is all easy to use. Visit http://www.dovepress.com/testimonials.php to read real quotes from published authors. 